\title{
Review
}

\section{Review of Research on the Relationship between Malassezia Infection and Psoriasis}

Yuan Liu

Department of Dermatovenereology, Tianjin Medical University General Hospital, Tianjin China

\author{
Keywords \\ psoriasis; Malassezia; relevance \\ Correspondence \\ Yuan Liu, \\ E-mail: yliutjmu@126.com
}

DOI: $10.1515 / i$ ii-2017-0132

\begin{abstract}
Psoriasis is a common clinical disease, and its pathogenesis is closely related to infecting microorganisms. Studies in recent years indicated that Malassezia plays an important role in occurrence and development of psoriasis. This study provides an overview on the role of Malassezia in development of psoriasis based on three aspects, namely, clinical observation, antifungal therapy, and immunology research.
\end{abstract}

Psoriasis is a common chronic inflammatory skin disease characterized by histopathological changes, such as excessive cell hyperplasia, infiltration of inflammatory cell, and neovascularization, in main tissues. Etiology of this disease remains unknown, and its pathogenesis is complex. Heredity, immunity, and environment play important roles in development of psoriasis. Information obtained both locally and abroad confirmed that psoriasis is associated with human leucocyte antigen (HLA). In recent years, Jianhua et al. ${ }^{[1]}$ also observed that HLA-DRB1*07 allele may be a susceptible gene among psoriasis vulgaris patients in Xinjiang Uygur Autonomous Region. Psoriasis is a T cell-mediated skin disease. The interaction between $\mathrm{T}$ cells and keratinocytes play a very critical role in psoriasis development. Further studies are still required to describe antigen stimulation that activates $\mathrm{T}$ cells. However, increasing clinical observation and experimental studies confirmed that microbial superantigens can activate $\mathrm{T}$ cells and induce psoriasis occurrence. Malassezia plays an important role in psoriasis pathogenesis and is the common symbiotic yeast found on human skin. This study provides an overview on the role of Malassezia in psoriasis development based on three aspects, namely, clinical observation, antifungal therapy, and immunological research.

\section{Clinical observation}

Malassezia is a kind of yeast and one of the resident organism in human skin, and it easily survives in the chest, back and scalp, and other body parts that are rich in sebaceous glands; this condition may be caused by insufficient synthesis of fatty acids by Malassezia and its need for exogenous fatty acids ${ }^{[2]}$. Psoriatic lesions are rich in loose-scale lipid content and coupled with long-term topical glucocorticoids. Psoriatic lesions are conducive for growth and reproduction of Malassezia. Malassezia isolated from psoriatic lesion causes more severe psoriasis in comparison with Malassezia that are obtained from other sources ${ }^{[3]}$. Japanese scholars used polymerase chain reaction to conduct quantitative analysis on DNA of various Malassezia samples obtained from psoriatic lesions of 20 psoriasis and healthy-skin patients and observed that colonization rate of restricted Malassezia on the body surface of psoriasis patients is five times higher than that of spherical Malassezia; thus, restricted Malassezia is the dominant species in psoriatic lesions ${ }^{[4]}$. Psoriasis can be expressed as early increases in dandruff, whereas spherical Malassezia and restricted Malassezia are the main predisposing factors of scalp condition ${ }^{[5,6]}$.

Studies showed that heat-inactivated oval Malassezia suspension may result in erythema and white scales on the back skins of rabbits, microscopic pathological changes in keratosis, dermal papillary capillary expansion and polymorphonuclear leukocyte infiltration, and other typical psoriasis changes ${ }^{[7]}$. Lober et al. ${ }^{[8]}$ used cell debris of Malassezia to perform patch test in forearms of psoriasis patients without psoriatic lesions. Results showed that 
all patients presented new psoriatic lesions in test sites. Narang et al. ${ }^{[9]}$ reported cases that Malassezia folliculitis and pityriasis versicolor lesions transform into guttiform psoriasis lesions. Thus, these researchers speculated that Malassezia plays a key role in psoriasis isomorphism reaction, further confirming the correlation between Malassezia and psoriasis.

Although correlation exists between Malassezia infection and psoriasis, full and direct clinical evidence remains inadequate. A large number of unexplored areas include the role of Malassezia in pathogenesis of different types of psoriasis, distribution, and pathogenesis of Malassezia in psoriatic lesions at different ages and normal skin lesions.

\section{Antifungal therapy}

Psoriasis patients with stubborn psoriasis are given clinical treatment, and clinical symptoms of psoriasis improve as skin maladies decrease. In 1982, Rosenberg et al. ${ }^{[7]}$ reported significant results achieved by using ketoconazole to treat scalp refractory psoriasis. Jie ${ }^{[10]}$ reported 49 cases of psoriasis patients with pityrosporum infection and treated with $0.1 \%$ haciendaide ointment, $2 \%$ ketone droplete lotion, and $2 \%$ ketoconazole cream each morning and evening for four weeks. These scientists observed that subjective symptoms decreased from the second week of treatment, skin lesions gradually diminished, scales decreased, and color faded or completely subsided. Curative effects were more significant higher than those of control group that only received $0.1 \%$ hacceptide ointment. Results suggest that good curative effects can be achieved by using antifungal drugs in patients with psoriasis vulgaris infected with Malassezia. Ketoconazole can improve psoriasis in seborrheic parts, and this result may be associated with direct inhibition of Malassezia growth or indirect inhibition of Malassezia-induced lymphocyte-mediated immune response ${ }^{[11]}$. In mainland China, ketoconazole and other antifungal drugs are listed as first-line drugs for psoriasis, but European psoriasis experts suggested that in scalp psoriasis classification and management consensus, ketoconazole can help patients with immunosuppressive diseases or combined Malassezia infection ${ }^{[12]}$.

Although ketoconazole poses curative effects in treatment of fungal infection, liver injury and other side effects frequently occur. Ketoconazole was gradually replaced by itraconazole. Itraconazole exhibits remarkable curative effects on pityriasis, Malassezia folliculitis, and other diseases. Itraconazole is safe, efficient, and economical. Ecaconazole can inhibit fungal growth by inhibiting ergosterol synthesis and presents antifungal effect by inducing host keratinocytes to produce human defensin ${ }^{[13]}$. Seborrheic dermatitis is also an inflammatory skin disease associated with Malassezia; itraconazole shock treatment effectively treats severe seborrheic dermatitis and can significantly improve clinical symptoms of patients ${ }^{[14]}$.

Antifungal agents are occasionally ineffective in treatment of psoriasis combined with Malassezia infection; such result may be caused by differing sensitivities of Malassezia subspecies to different antifungal agents. Studies ${ }^{[15]}$ showed significantly higher sensitivities of $M$. sympodialis and $M$. pachydermatis to ketoconazole and itraconazole than $M$. furfur, M. globosa, M. sloofiae, and restricted Malassezia. This finding advises that when using antifungal agents to treat Malassezia-infected psoriasis with poor efficacy, Malassezia drug-sensitivity tests should be conducted, and sensitive antifungal agents must be utilized to achieve good curative effects. Early use of antifungal agents in treatment of scalp psoriasis combined with Malassezia infection achieved satisfactory results; however, these studies lacked depth and require further confirmation ${ }^{[4]}$. No report is available on the use of new antifungal drugs, such as leverconazole and posaconazole, in treatment of psoriasis infected by Malassezia.

\section{Immunological studies}

Malassezia can parasitize the skin and cause psoriasis and other inflammatory diseases. A complex immune mechanism results in such dual biological behavior. In normal skin, Malassezia downregulates inflammatory reaction of hosts by inducing transforming growth factor- $\beta 1$ and interleukin (IL). IL-10 downregulates phagocytosis of monocytes by inhibiting IL-1, IL-6, and tumor necrosis factor- $\alpha$ (TNF- $\alpha$ ) to achieve long-term symbiosis on host skin ${ }^{[16,17]}$. Malassezia also interacts with defective skin barrier to exacerbate psoriasis by promoting inflammatory responses and accelerating hyperproliferation of epithelial cell.

Some scholars suggested that psoriasis is a helper $\mathrm{T}$ lymphocyte (Th). Th1/Th2 imbalance of autoimmune disease, mucosal and overexpression of proinflammatory effect of Th1 cytokines, and low expression of antiinflammatory effect of Th2 cytokines play certain roles 
in occurrence, maintenance, and recurrence of psoriasis; imbalance of inflammatory media and cytokine network also plays an important role in chronic plaque psoriasis ${ }^{[18]}$. Malassezia can induce occurrence and deterioration of psoriasis through differentiation of Th cell subsets in patients with psoriasis. Hydrophobic components of Malassezia can stimulate expression of ribonucleic acid of proinflammatory factor messenger in human epidermal keratinocytes; thus, Th subsets in peripheral blood show skewness toward Th1 cell differentiation ${ }^{[19]}$. Levels of IL-4, IL-10, IL-13, and other Th2 cytokines are significantly lower in sera of patients with Malassezia colonization-positive psoriasis guttata than patients with Malassezia colonization-negative psoriasis guttata. Expression of downregulation of Th2 cytokines in Malassezia is related to occurrence of psoriasis guttata ${ }^{[20]}$.

Psoriasis vulgaris lesions present remarkable lymphocyte and mononuclear cell infiltration. IL-1, IL-6, interferon- $\gamma$ (IFN- $\gamma$ ), and other cytokines released by $\mathrm{T}$ cells can stimulate proliferation of keratinocytes to shorten epidermal replacement time. IFN- $\gamma$ can also induce signal transduction and transcriptional activator 3 phosphorylation, activate its downstream genes, stimulate proliferation of keratinocytes, and promote and participate in progress of psoriasis ${ }^{[21]}$. The complex interaction between Malassezia and keratinocytes play an important role in occurrence of psoriasis. Malasseziaderived soluble components exhibit chemotactic effects on polymorphonuclear leukocytes of psoriasis patients and can stimulate proliferation of keratinocytes through intracellular Toll-like receptors or Nod-like signaling pathways ${ }^{[22]}$. Host immune system can recognize lipophilic components and hydrophilic components in Malassezia, induce IL-8 release, activate the complement system, and induce migration of host immune cells to the dermis ${ }^{[23]}$ through macrophageinduced C-type lectin-like receptors and dendrite cellassociated lectin 2 .

Malassezia cell wall contains abundant lipid components, which are key components that affect host immune responses to Malassezia. Coculture of Malassezia without cell wall and keratinocytes showed that keratinocytes IL-6, IL-8, and IL-1 $\alpha$ significantly increased, whereas IL-10 significantly decreased; thus, lipid composition of Malassezia may regulate production of cytokines through keratinocytes ${ }^{[24]}$. Kim et al. ${ }^{[25]}$ observed similar morphologies of detergenttreated Malassezia and Malassezia without lipid components. However, detergent-treated Malassezia can induce mononuclear cells to produce more TNF- $\alpha$.

\section{Conclusion}

In summary, although clinical observations, antifungal therapies, and immunological studies showed that a correlation exists between Malassezia infection and occurrence and development of psoriasis, a large number of unknown fields require additional in-depth studies. Researchers must explore the mechanism of antifungal agents in treatment of psoriasis combined with Malassezia infection from a therapeutic point of view or further investigate pathogenic mechanism that triggers Malassezia infection or aggravate psoriasis from the immunological point of view.

\section{Declarations}

\section{Acknowledgements}

No.

\section{Competing interests}

The author declares that he has no competing interest.

\section{Authors' contributions}

Y Liu made the literature analysis and wrote, discussed and revised the manuscript of this review.

\section{References}

1 Jiang J, Duo L. Study on the Correlation between Psoriasis Vulgaris and HLADRB1*07 allele in Xinjiang Uygur Autonomous Region. Journal of Medical Postgraduates, 2012, 25 (1): 54-57.

2 Hort W, Mayser P. Malassezia virulence determinants. Curr Opin Infect Dis, 2011, 24(2): 100-105.

3 Rup E, Skora M, Krzysciak P, et al. Distribution of Malassezia species in patients with psoriasis-quality assessment. Postep Dermatol Alergol, 2010, 27(4): 264-268.

4 Gaitanis G, Magiatia P, Hantschke M, et al. The Malassezia genus in skin and systemic diseases. Clin Microbiol Rev, 2012, 25(1): 106-141.

5 James AG, Abraham KH, Cox DS, et al. Metabolic analysis of the cutaneous fungi Malassezia globosa and M. restricta for insights on scalp condition and dandruff. Int J Cosmet Sci, 2013, 35(2): 169-175.

6 Kim D, Lim YR, Ohk SO, et al. Functional expression and characterization of CYP51 from dandruff-causing Malassezia globosa. FEMS Yeast Res, 2011, 11(1): 80-87.

7 Rosenberg EW, Belew P, Bale G. Effct of topical application of heavy suspensions of killed Malassezia Ovalis on rabbit skin.Mycopathologia, 
1980, $72(3): 147-154$.

8 Lober CW, Rosenberg EW, Bale G. Patch test with killed sonicated microflora in patients with psoriasis. Arch Dermatol, 1982, 118(5): 322-325.

9 Narang T, Dogra S, Kaur I, et al. Malassezia and psoriasis: Koebner's phenomenon or direct causation? J Eur Acad Dermatol Venereol, 2007, 21(8): 1111-1112.

10 Zhang J. Clinical Observation on 96 Cases of Psoriasis Vulgaris combined with Pityrosporum Infection. Journal of Nantong University (Medical Sciences), 2005, 25 (2): 129.

11 Alford RH, Vire CG, Cartwright BB, et al. Ketoconazole s Inhibition of Fungal Antigen-Induced Thymidine Uptake by Lymphocytes from Patients with Psoriasis. Am J Med Sci, 1986, 2(291): 75-80.

12 Ortonne J, Chimenti S, Luger T, et al. Scalp psoriasis: European consensus on grading and treatment algorithm. J Eur Acad Dermatol Venereol, 2009, 23(12): 1435-1444.

13 Kanda N, Kano R, Ishikawa T, et al. The antimycotic drugs itraconazole and terbinafine hydrochloride induce the production of human $\beta$-defensin-3 in human keratinocytes. Immunobiology, 2011, 216(4): 497-504.

14 Trznadel-Grodzka E, Btaszkowski M, Rotsztejn H. Investigations of seborrheic dermatitis. Part II. Influence of itraconazole on the clinical condition and the level of selected cytokines in seborrheic dermatitis. Postepy Hig Med Dosw (Online), 2012, 66:848-854.

15 Diskshit A, Tiwari AK, Mishra RK. New Mediun for Rapid Diagnosis and Determination of Antifungal Testing Against Malassezia spp: A Potential Candidate for Industries. Natl Acad Sci Lett, 2013, 36(1): 61-66. Akaza N, Akamatsu H, Takeoka S, et al. Increased hydrophobicity in Malassezia species correlates with increased proinflammatory cytokine expression in human keratinocytes. Med Mycol, 2012, 50(8): 802-810.
17 Buommino E, De Filippis A, Parisi A, et al. Innate immune response in human keratinocytes infection by a feline isolate of Malassezia pachydermatis. Vet Microbiol, 2013, 163(1-2): 90-96.

18 Swindell WR, Xing X, Stuart PE, et al. Heterogeneity of inflammatory and cytokine networks in chronic plaque psoriasis. PLoS One, 2012, 7(3): e34594.

19 Akaza N, Akamatsu H, Takeoka S, et al. Increased hydrophobicity in Malassezia species correlates with increased proinflammatory cytokine expression in human keratinocyte. Med Mycol, 2012, 50(8): 802-810.

20 Aydogan K, Tore O, Akcaglar S, et al. Effects of Malassezia yeasts on serum Th1 and Th 2 cytokines in patients with guttate psoriasis. Int $\mathrm{J}$ Dermatol, 2013, 52(1): 46-52.

21 Feng S, Wei J, Han L. Review of Research on Signal Transduction and Transcriptional Activator 3 Signaling Pathway in the Pathogenesis and Treatment of Psoriasis. Journal of Medical Postgraduates, 2013, 26 (4): 416-420.

22 Mattozzi C, Richetta AG, Cantisani C, et al. Psoriasis: New insight about pathogenesis, role of barrier organ integrity, NLR/CATERPILLER family genes and microbial flora. J Dermatol, 2012, 39(9): 752-760.

23 Ishikawa $\mathrm{T}$, Itoh F, Yoshida $\mathrm{S}$, et al. Identification of Distinct Ligands for the C-type Lectin Receptors Mincle and Dectin-2 in the Pathogenic Fungus Malassezia. Cell Host Microbe, 2013, 13(4): 477-488.

24 Thomas DS, Ingham E, Bojar RA, et al. In vitro modulation of human keratinocyte pro- and anti-inflammatory cytokine production by the capsule of Malassezia species. FEMS Immunol Med Microbiol, 2008, 54(2): 203-214.

$25 \mathrm{Kim} \mathrm{SH}, \mathrm{Ko} \mathrm{HC}$, Kim MB, et al. The effect of detergents on the morphology and immunomodulatory activity of Malassezia furfur. Ann Dermatol, 2009, 21(2): 130-135. 